\title{
Renal safety and urate-lowering efficacy of febuxostat in gout patients with stage $4-5$ chronic kidney disease not yet on dialysis
}

\author{
Sang-Hyon Kim*, So-Yeon Lee*, Ji-Min Kim, and Chang-Nam Son
}

Division of Rheumatology, Department of Internal Medicine, Keimyung University School of Medicine, Daegu, Korea

\author{
Received: November 25, 2018 \\ Revised : February 22, 2019 \\ Accepted: March 12, 2019

\section{Correspondence to} \\ Chang-Nam Son, M.D. \\ Division of Rheumatology, \\ Department of Internal \\ Medicine, Keimyung University \\ School of Medicine, 56 \\ Dalseong-ro, Jung-gu, Daegu \\ 41931, Korea \\ Tel: +82-53-250-7611 \\ Fax: +82-53-250-7434 \\ E-mail: cnson@kmu.ac.kr
}

*These authors contributed equally to this work.
Background/Aims: The safety and efficacy of febuxostat in patients with stage 4-5 chronic kidney disease (CKD) are still unclear owing to a lack of studies in these patients. Therefore, we aimed to evaluate the effect of febuxostat on renal function, general safety, and efficacy in gout patients with stage 4-5 CKD.

Methods: Among 739 patients who had been administered febuxostat from May 2012 to December 2016 at a single hospital in Korea, 370 patients who had been monitored for 1 year were analyzed. Serum uric acid levels and estimated glomerular filtration rate (eGFR) of patients with gouty arthritis were collected at baseline and 1 year after febuxostat administration.

Results: Among the 370 patients, 280 patients were stage 1-3 CKD, 63 patients were stage 4-5 CKD, and 27 patients were on dialysis. The eGFR of 63 patients with stage 4-5 CKD, excluding dialysis patients, was $19.84 \pm 7.08 \mathrm{~mL} / \mathrm{min} / 1.73$ $\mathrm{m}^{2}$ when they began to take febuxostat and $23.49 \pm 16.67 \mathrm{~mL} / \mathrm{min} / 1.73 \mathrm{~m}^{2}$ after 12 months $(p=0.13)$. The urate-lowering effect after 12 months of febuxostat medication showed statistical significance $(8.96 \pm 2.31 \mathrm{mg} / \mathrm{dL}$ at baseline and $4.88 \pm 1.68$ $\mathrm{mg} / \mathrm{dL}$ after 12 months, $p<0.01)$. The difference in incidence of adverse events among patients with stage 1-3 CKD, those with stage 4-5 CKD, and those on dialysis was not significant.

Conclusions: Febuxostat demonstrated renal safety and good urate-lowering efficacy in gout patients with stage $4-5 \mathrm{CKD}$, who are not yet on dialysis.

Keywords: Febuxostat; Gout; Renal insufficiency, chronic

\section{INTRODUCTION}

Gout is a rheumatic disease resulting from the deposition of monosodium urate crystal in joint fluid or soft tissues due to increased levels of serum uric acid (SUA) [1]. Hyperuricemia is caused by an imbalance between uric acid production and excretion. It not only results in gout and kidney failure, but is also a main cause of hypertension and hyperlipidemia. Chronic kidney disease (CKD) causes hyperuricemia, which is a main cause of gout [2]. Previous studies reported that patients with CKD accounted for $39 \%$ of patients with gout [3]. Thus, there has been an increasing interest in lifestyle modifications and pharmacological treatment among patients with hyperuricemia.

Gout management guidelines by the European League Against Rheumatism (EULAR) recommend allopurinol, which is metabolized by the kidney, as a first-line urate-lowering drug. As oxypurinol, an active metabolite of allopurinol, can cause several side effects in patients 


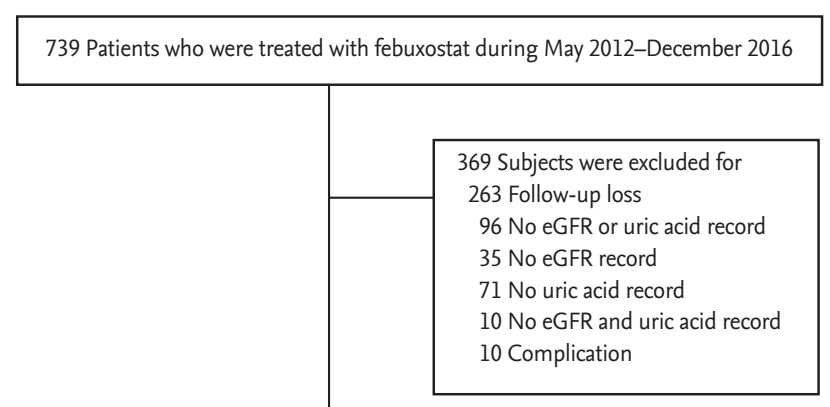

370 Patients who were treated with febuxostat for 12 months

63 eGFR < $30 \mathrm{~mL} / \mathrm{min} / 1.73 \mathrm{~m}^{2}:$ CKD stage 4 , 5 , excluding patients on dialysis

27 Patients on dialysis

Figure 1. Flow chart illustrating inclusion and exclusion criteria for the study subjects. eGFR, estimated glomerular filtration rate; CKD, chronic kidney disease.

with CKD, allopurinol dosage should be lowered in patients with CKD depending on their kidney function [4]. In contrast, febuxostat, another urate-lowering agent, is metabolized by the liver. Febuxostat has advantages over allopurinol in that there is no dosage limit associated with renal condition in patients with stage 1-3 CKD [5].

However, as phase 3 clinical trials do not involve patients with stage $4-5$ chronic kidney failure, whose glomerular filtration rate is below $30 \mathrm{~mL} / \mathrm{min} / 1.73 \mathrm{~m}^{2}$, the safety and efficacy of febuxostat is unclear in the aforementioned patients [6]. This study aims to examine the efficacy and safety of 12-month administration of febuxostat in patients with stage $4-5$ CKD.

\section{METHODS}

\section{Patients}

Among 739 in- and outpatients who had been administered febuxostat from May 2012 to December 2016 at a university teaching hospital, 370 patients who had been monitored for 12 months (9 to 15 months) and had their SUA levels and estimated glomerular filtration rate (eGFR) checked before and after taking febuxostat were enrolled in the study. The following patients were excluded: 263 patients who were not monitored during the 12-month period; 96 patients who did not have their glomerular filtration rates and uric acid levels checked during the 1-year monitoring; and 10 patients who discontinued febuxostat due to side effects (Fig. 1). This study was conducted under the review of the Institutional Review Board at Keimyung University Hospital (IRB no: 2017-01-021). As this is a retrospective study, the need for consent was waived.

\section{Study design}

A survey on the basic information (age, gender), SUA, eGFR, serum creatinine levels, comorbidities, types of preventive treatment, febuxostat dosage, and types of urate-lowering agents taken previously was conducted in the enrolled patients $(n=370)$ at the time of febuxostat initiation. In addition, their SUA levels and eGFR after 12 months (9 to 15 months) of taking febuxostat were determined. eGFR were calculated using the Modification of Diet in Renal Disease based on serum creatinine levels [7].

\section{Statistical analysis}

Results are presented as mean \pm SD unless specified otherwise. The Kruskal-Wallis test was used for between-group comparisons. Fisher's exact test was used to assess the frequencies of gender, underlying disease, types of preventive treatment, and types of urate-lowering agents taken previously in between-group comparisons. A paired $t$ test was conducted to identify changes in SUA levels and eGFR. SPSS for window version 20.0 (IBM Co., Armonk, NY, USA) was used for statistical analysis. A $p$ value less than 0.05 was considered significant.

\section{RESULTS}

\section{Baseline characteristics of enrolled subjects}

Among a total of 370 patients, Sixty-three patients (mean age, $63.7 \pm 12.4$ years; male to female ratio, 41:22) had stage $4-5$ CKD (Table 1). Mean \pm SD serum creatinine and SUA were $3.6 \pm 2.6,9.0 \pm 2.3$, respectively. Regarding comorbidities, 47 had hypertension (74.6\%) and 25 had diabetes (39.7\%). Preventive treatment was given to 21 patients. Among them, 14 had taken steroids (22.2\%), which accounted for the highest number. Regarding the dosage of febuxostat, 48 patients took $40 \mathrm{mg}(76.2 \%)$, accounting for the highest number of patients. Regarding 
Table 1. Demographic and clinical characteristics of patients

\begin{tabular}{|c|c|c|c|c|c|}
\hline \multirow[b]{3}{*}{ Characteristic } & \multirow{3}{*}{$\begin{array}{l}\text { All patients } \\
\quad(\mathrm{n}=370)\end{array}$} & \multicolumn{3}{|c|}{ Baseline eGFR, $\mathrm{mL} / \mathrm{min} / 1.73 \mathrm{~m}^{2}$} & \multirow[b]{3}{*}{$p$ value } \\
\hline & & \multirow{2}{*}{$\begin{array}{c}\text { eGFR } \geq 30 \\
\text { Except patients } \\
\text { on dialysis } \\
(\mathrm{n}=28 \mathrm{o})\end{array}$} & \multicolumn{2}{|c|}{ eGFR $<30$} & \\
\hline & & & $\begin{array}{c}\text { Except patients } \\
\text { on dialysis } \\
(\mathrm{n}=63)\end{array}$ & $\begin{array}{c}\text { Patients } \\
\text { on dialysis } \\
(\mathrm{n}=27)\end{array}$ & \\
\hline Age, yr & $60.49 \pm 13.30$ & $59.66 \pm 13.50$ & $63.70 \pm 12.43$ & $61.70 \pm 12.43$ & NS \\
\hline Creatinine, mg/dL & $1.94 \pm 2.14$ & $1.18 \pm 0.40$ & $3.60 \pm 2.64$ & $5.92 \pm 4.15$ & $<0.01$ \\
\hline \multicolumn{6}{|l|}{ Sex } \\
\hline Male & $324(87.6)$ & $262(93.6)$ & $41(65.1)$ & $21(77.8)$ & $<0.01$ \\
\hline Female & $46(12.4)$ & $18(6.4)$ & $22(34.9)$ & $6(22.2)$ & \\
\hline \multicolumn{6}{|l|}{ Serum uric acid, mg/dL } \\
\hline Mean \pm SD & $8.13 \pm 2.24$ & $7.98 \pm 2.06$ & $8.96 \pm 2.31$ & $7.87 \pm 3.33$ & NS \\
\hline \multicolumn{6}{|l|}{ Range } \\
\hline$<7$ & $100(27.0)$ & $78(27.9)$ & $10(15 \cdot 9)$ & $12(44 \cdot 4)$ & \\
\hline $7-8$ & $67(18.1)$ & $58(20.7)$ & $6(9 \cdot 5)$ & $3(11.1)$ & \\
\hline $8-9$ & $89(24.1)$ & $71(25.4)$ & $16(25.4)$ & $2(7.4)$ & \\
\hline$\geq 9$ & $114(30.8)$ & $73(26.1)$ & $31(49.2)$ & $10(37.0)$ & \\
\hline \multicolumn{6}{|l|}{ Comorbidity $^{\mathrm{a}}$} \\
\hline $\mathrm{DM}$ & $94(25 \cdot 4)$ & $58(20.7)$ & $25(39.7)$ & $11(40.7)$ & $<0.01$ \\
\hline HTN & $204(55.1)$ & $140(50.0)$ & $47(74.6)$ & $17(63.0)$ & $<0.01$ \\
\hline CKD & $181(48.9)$ & $96(34 \cdot 3)$ & $60(95.2)$ & $25(92.6)$ & $<0.01$ \\
\hline CAD & $59(15 \cdot 9)$ & $43(15 \cdot 4)$ & $14(22.2)$ & $2(7 \cdot 4)$ & NS \\
\hline CVA & $40(10.8)$ & $30(10.7)$ & $8(12.7)$ & $2(7.4)$ & NS \\
\hline Liver disease & $12(3.2)$ & $6(2.1)$ & $4(6.3)$ & $2(7.4)$ & NS \\
\hline Cancer & $14(3.8)$ & $11(3.9)$ & $3(4.8)$ & 0 & NS \\
\hline \multicolumn{6}{|l|}{ Prophylactic therapy } \\
\hline No therapy & $161(43.5)$ & $101(36.1)$ & $42(66.7)$ & $18(66.7)$ & \\
\hline Steroid & $68(18.4)$ & $50(17.9)$ & $14(22.2)$ & $4(14.8)$ & \\
\hline Colchicine & $103(27.8)$ & $95(33.9)$ & $5(7.9)$ & $3(11.1)$ & \\
\hline NSAIDs & $18(4 \cdot 9)$ & $15(5 \cdot 4)$ & $1(1.6)$ & $2(7 \cdot 4)$ & \\
\hline Steroid/colchicine & $14(3.8)$ & $13(4.6)$ & $1(1.6)$ & 0 & \\
\hline NSAIDs/colchicine & $6(1.6)$ & $6(2.1)$ & $\mathrm{O}$ & $\mathrm{O}$ & \\
\hline \multicolumn{6}{|l|}{ Dose of febuxostat, mg } \\
\hline 40 & $243(65 \cdot 7)$ & $171(61.1)$ & $52(82.5)$ & $24(88.9)$ & $<0.05$ \\
\hline 60 & $44(11.9)$ & $37(13.2)$ & $1(1.6)$ & $2(7 \cdot 4)$ & \\
\hline 80 & $83(22.4)$ & $72(25 \cdot 7)$ & $10(15 \cdot 9)$ & $1(3.7)$ & \\
\hline \multicolumn{6}{|l|}{ Previous urate-lowering treatment } \\
\hline Allopurinol & $237(64.1)$ & $191(68.2)$ & $48(76.2)$ & $12(44 \cdot 4)$ & $<0.01$ \\
\hline Benzbromarone & $7(1.9)$ & $6(2.1)$ & $5(7 \cdot 9)$ & o & \\
\hline Unknown & $12(3.2)$ & $9(3.2)$ & $10(15 \cdot 9)$ & $2(7 \cdot 4)$ & \\
\hline No previous ULT & $89(24.1)$ & $62(22.1)$ & $16(25.4)$ & $11(40.7)$ & \\
\hline Allopurinol/benzbromarone & $25(6.8)$ & $12(4 \cdot 3)$ & $11(17 \cdot 5)$ & $2(7 \cdot 4)$ & \\
\hline
\end{tabular}

Values are presented as mean \pm SD or number (\%).

eGFR, estimated glomerular filtration rate; NS, not significant; SD, standard deviation; DM, diabetes mellitus; HTN, hypertension; CKD, chronic kidney disease; CAD, coronary artery disease; CVA, cerebrovascular accident; NSAID, nonsteroidal anti-inflammatory drug; ULT, uric acid-lowering treatment.

aTotal subjects $(n=287):$ GFR $\geq 30 \mathrm{~mL} / \mathrm{min} / 1.73 \mathrm{~m}^{2}(\mathrm{n}=197)$, eGFR < $30 \mathrm{~mL} / \mathrm{min} / 1.73 \mathrm{~m}^{2}(\mathrm{n}=63)$, patients on dialysis $(\mathrm{n}=27)$. 
A

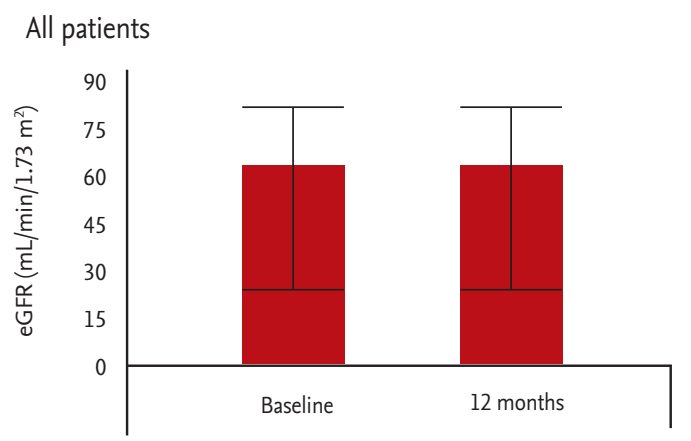

$\mathrm{eGFR} \geq 30 \mathrm{~mL} / \mathrm{min} / 1.73 \mathrm{~m}^{2}$

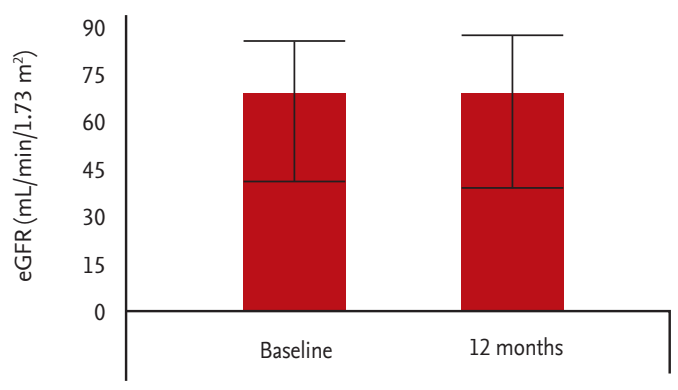

eGFR $<30 \mathrm{~mL} / \mathrm{min} / 1.73 \mathrm{~m}^{2}$

$\mathbf{E}$

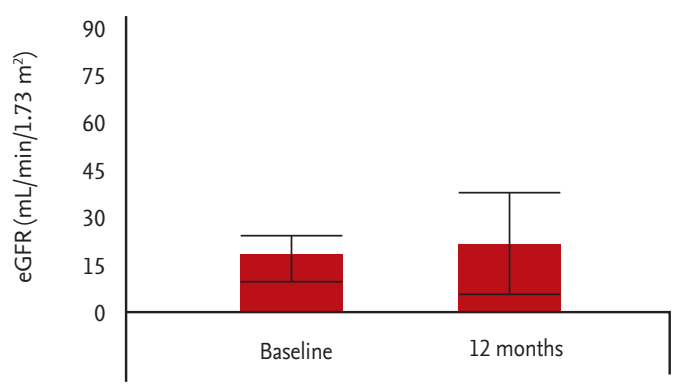

B

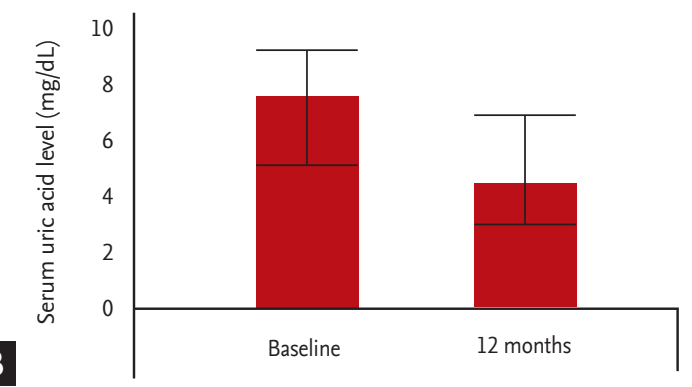

D

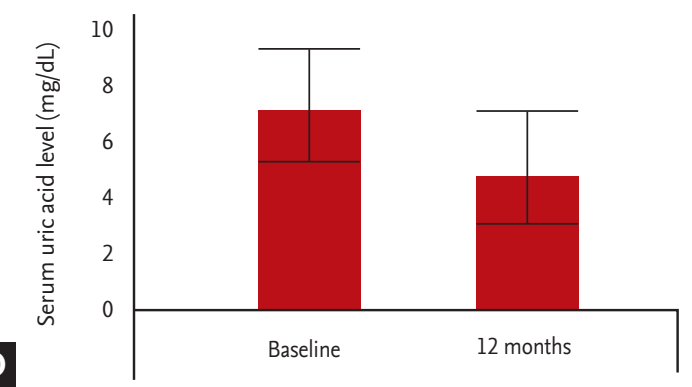

$\mathbf{F}$

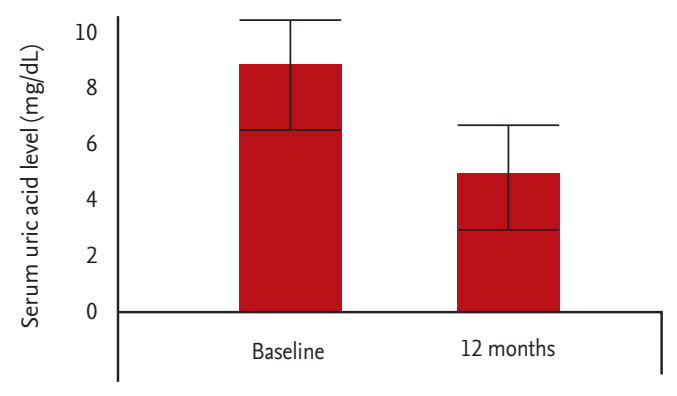

Figure 2. Changes in estimated glomerular filtration rate (eGFR) and serum uric acid levels after 12 months of febuxostat medication. (A) All patients $(\mathrm{n}=37 \mathrm{O})$. (B) Baseline eGFR $\geq 30 \mathrm{~mL} / \mathrm{min} / 1.73 \mathrm{~m}^{2}$, and not under dialysis therapy $(\mathrm{n}=28 \mathrm{o})$. (C) Baseline eGFR $<30 \mathrm{~mL} / \mathrm{min} / 1.73 \mathrm{~m}^{2}$, and not under dialysis therapy $(\mathrm{n}=63)$.

the types of urate-lowering agents used previously, 34 patients took allopurinol (54.0\%), which accounted for the largest percentage.

\section{Safety of febuxostat}

The eGFR of 63 patients with stage 4-5 CKD, excluding dialysis patients, were $19.84 \pm 7.08 \mathrm{~mL} / \mathrm{min} / 1.73 \mathrm{~m}^{2}$ when they began to take febuxostat, and $23.49 \pm 16.67$ $\mathrm{mL} / \mathrm{min} / 1.73 \mathrm{~m}^{2}$ after 12 months (Fig. 2). There was no significant difference in eGFR before taking febuxostat and after 12 months in all the three groups (all patients, stage 1-3 CKD, and stage 4-5 CKD, respectively). Regarding 49 patients with stage 4 CKD excluding dialysis patients, the eGFR were $22.75 \pm 4.80 \mathrm{~mL} / \mathrm{min} / 1.73 \mathrm{~m}^{2}$ before taking febuxostat and $22.49 \pm 7.93 \mathrm{~mL} / \mathrm{min} / 1.73 \mathrm{~m}^{2}$ after taking febuxostat $(p=0.82)$. In 14 patients with stage 5 $\mathrm{CKD}$, the eGFR were $9.65 \pm 3.17 \mathrm{~mL} / \mathrm{min} / 1.73 \mathrm{~m}^{2}$ before taking febuxostat and $27.01 \pm 32.79 \mathrm{~mL} / \mathrm{min} / 1.73 \mathrm{~m}^{2}$ after 
Table 2. Febuxostat complications

\begin{tabular}{lccc}
\hline Febuxostat complications & $\begin{array}{c}\text { eGFR } \geq 30 \mathrm{~mL} / \mathrm{min} / 1.73 \mathrm{~m}^{2} \\
\text { Except patients on dialysis } \\
(\mathrm{n}=516)\end{array}$ & $\begin{array}{c}\text { eGFR }<30 \mathrm{~mL} / \mathrm{min} / 1.73 \mathrm{~m}^{2} \\
\text { Except patients on dialysis } \\
(\mathrm{n}=109)\end{array}$ & $\begin{array}{c}\text { Patients on } \\
\text { dialysis } \\
(\mathrm{n}=49)\end{array}$ \\
\hline Total number & 15 & 3 & 2 \\
Diarrhea & $4(26.7)$ & 0 & $2(100.0)$ \\
Dermatitis & $6(40.0)$ & 0 & 0 \\
Musculoskeletal pain and discomfort & $2(13.3)$ & $2(66.7)$ & 0 \\
LFT rising $<3 \times \mathrm{UNL}$ & $2(13.3)$ & $1(33.3)$ & 0 \\
LFT rising $\geq 3 \times \mathrm{UNL}$ & $1(6.7)$ & 0 & 0 \\
\hline
\end{tabular}

Values are presented as number (\%).

eGFR, estimated glomerular filtration rate; LFT, liver function test; UNL, upper normal limit.

12 months $(p=0.43)$. In stage $4-5$ CKD, adverse events occurred two cases $(66.7 \%)$ of myalgia and one case of elevated liver function (33.3\%) (Table 2).

\section{Efficacy of febuxostat}

In 63 patients with stage $4-5$ CKD excluding dialysis, the SUA levels were $8.96 \pm 2.31 \mathrm{mg} / \mathrm{dL}$ when they began to take febuxostat and $4.88 \pm 1.68 \mathrm{mg} / \mathrm{dL}$ after 12 months (Fig. 2). In 49 patients with stage 4 CKD excluding dialysis, the SUA levels were $8.78 \pm 2.22 \mathrm{mg} / \mathrm{dL}$ before taking febuxostat and $5.04 \pm 1.69 \mathrm{mg} / \mathrm{dL}$ after febuxostat administration $(p<0.01)$. In 14 patients with stage $5 \mathrm{CKD}$, the SUA levels were $9.59 \pm 2.59$ and $4.29 \pm 1.58 \mathrm{mg} / \mathrm{dL}$ after 12 months $(p<0.01)$. SUA levels were reduced significantly after 12 months of febuxostat administration in all the three groups (all patients, stage 1-3 CKD, and stage 4-5 CKD, respectively).

\section{DISCUSSION}

In this study, we aimed to determine the effect of febuxostat on renal function, safety, and efficacy in gout patients with stage $4-5 \mathrm{CKD}$, and found that febuxostat demonstrated renal safety and good urate-lowering efficacy in these patients.

Uric acid is the final metabolite of purine, and approximately $70 \%$ of uric acid is excreted in urine. The prevalence of hyperuricemia and gout in patients with CKD is high owing to the reduction in urinary excretion of uric acid. The accumulation of uric acid occurs as a complication of renal failure. It also causes damage in the kidney, which can result in deterioration of renal failure, hypertension, and metabolic syndrome [2].

Although this study was retrospective analysis, owing to a lack of studies of patients with stage 4-5 CKD, it seemed to be valuable. The SUA levels of $\sigma_{3}$ patients with stage $4-5 \mathrm{CKD}$ excluding dialysis were reduced to 4.88 from $8.96 \mathrm{mg} / \mathrm{dL}$. This finding indicates that febuxostat is effective for patients with stage $4-5$ CKD. Omori et al. [8] reported that febuxostat reduced renal tubular damage and oxidation, thereby preventing endotheliocyte damage. Thus, febuxostat not only plays a role in efficiently lowering SUA levels in CKD patients, but is also considered an effective agent to prevent deterioration of renal function [9].

Stage 4-5 CKD patients had significant difference in eGFR before and after febuxostat administration. Sakai et al. [10] reported that febuxostat was effective in patients with CKD having hyperuricemia who did not respond to allopurinol treatment. In addition, eGFR increased with febuxostat. Tsuruta et al. [11] conducted a 1-year retrospective study of 73 patients with hyperuricemia, and found that the reduction of eGFR was slower in patients who were administered febuxostat than in those who had taken allopurinol.

There are certain limitations to this study. First, being a retrospective study based on medical records, there is a possibility that clinical factors may have been overlooked or underestimated. Second, since the subjects were 370 patients who had been treated at a single institution, the result of the study does not represent all pa- 
tients with gout. Third, the patients had comorbidities, such as hypertension, diabetes, cardiac and cerebrovascular diseases, and they had taken different medications before febuxostat administration. Considering these limitations, it is difficult to conclude that changes in SUA levels and eGFR are attributed to a single variable, i.e., febuxostat. Future studies on CKD patients should ensure that the patients have taken the same drug before febuxostat administration to obtain more significant research outcomes.

In summary, we found that febuxostat demonstrated renal safety and good urate-lowering efficacy in gout patients with stage $4-5 \mathrm{CKD}$.

\section{KEY MESSAGE}

1. This is a retrospective, observational study conducted at a single Korean hospital.

2. Febuxostat showed renal safety and good uratelowering efficacy in gout patients with stage $4-5$ chronic kidney disease.

\section{Conflict of interest}

No potential conflict of interest relevant to this article was reported.

\section{Acknowledgments}

This research was supported by the Keimyung University Research Grant of 2016

\section{REFERENCES}

1. Richette P, Bardin T. Gout. Lancet 2010;375:318-328.

2. Cirillo P, Sato W, Reungjui S, et al. Uric acid, the metabolic syndrome, and renal disease. J Am Soc Nephrol
2006;17:S165-S168.

3. Fuldeore MJ, Riedel AA, Zarotsky V, Pandya BJ, Dabbous O, Krishnan E. Chronic kidney disease in gout in a managed care setting. BMC Nephrol 2011;12:36.

4. Richette P, Doherty M, Pascual E, et al. 2016 Updated EULAR evidence-based recommendations for the management of gout. Ann Rheum Dis 2017;76:29-42.

5. Hira D, Chisaki Y, Noda S, et al. Population pharmacokinetics and therapeutic efficacy of febuxostat in patients with severe renal impairment. Pharmacology 2015;96:9098.

6. Schumacher HR Jr, Becker MA, Wortmann RL, et al. Effects of febuxostat versus allopurinol and placebo in reducing serum urate in subjects with hyperuricemia and gout: a 28-week, phase III, randomized, double-blind, parallel-group trial. Arthritis Rheum 2008;59:1540-1548.

7. Levey AS, Bosch JP, Lewis JB, Greene T, Rogers N, Roth D. A more accurate method to estimate glomerular filtration rate from serum creatinine: a new prediction equation. Modification of Diet in Renal Disease Study Group. Ann Intern Med 1999;130:461-470.

8. Omori H, Kawada N, Inoue K, et al. Use of xanthine oxidase inhibitor febuxostat inhibits renal interstitial inflammation and fibrosis in unilateral ureteral obstructive nephropathy. Clin Exp Nephrol 2012;16:549-556.

9. Keenan RT, O'Brien WR, Lee KH, et al. Prevalence of contraindications and prescription of pharmacologic therapies for gout. Am J Med 2011;124:155-163.

10. Sakai Y, Otsuka T, Ohno D, Murasawa T, Sato N, Tsuruoka

S. Febuxostat for treating allopurinol-resistant hyperuricemia in patients with chronic kidney disease. Ren Fail 2014;36:225-231.

11. Tsuruta Y, Mochizuki T, Moriyama T, et al. Switching from allopurinol to febuxostat for the treatment of hyperuricemia and renal function in patients with chronic kidney disease. Clin Rheumatol 2014;33:1643-1648. 